\title{
Cpvma1, a Vacuolar H+-ATPase Catalytic Subunit of Cryphonectria parasitica, is Essential for Virulence and Hypovirus RNA Accumulation
}

\author{
Ru Li, ${ }^{1,2}$ Shan Bai, ${ }^{2}$ Yuanyuan He,2 Qi Chen, ${ }^{2}$ Yanping Yao, ${ }^{2}$ Jinzi Wang, ${ }^{2}$ and Baoshan Chen ${ }^{1,2, \dagger}$ \\ ${ }^{1}$ State Key Laboratory for Conservation and Utilization of Subtropical Agro-Bioresources, Guangxi University, Nanning 530004, China \\ ${ }^{2}$ College of Life Science and Technology, Guangxi University, Nanning 530004, China \\ Accepted for publication 8 March 2019.
}

\begin{abstract}
The vacuolar $\mathrm{H}^{+}$-ATPases (V-ATPases) are conserved ATP-dependent proton pumps that acidify intracellular compartments in eukaryotic cells. The role of Cpvma1, a V-ATPase catalytic subunit A of Cryphonectria parasitica, was investigated by generating cpvmal-overexpressing and cpvmal-silenced strains. The mutant strains were evaluated for phenotypic characteristics, V-ATPase activity, response to elevated $\mathrm{pH}$ and $\mathrm{Ca}^{2+}$ in the medium, virulence on chestnut, and accumulation of hypovirus RNA in the cells. Compared with the wild-type strain, cpvmal-overexpressing strains showed no significant difference in phenotype; however, cpvmal-silenced

strains exhibited a phenotype of reduced growth rate, lower level of sporulation, and a marked decrease in V-ATPase activity and virulence. In addition, silencing of cpvmal increased sensitivity to elevated $\mathrm{pH}$ and $\mathrm{Ca}^{2+}$, implicating an important role for Cpvmal in $\mathrm{pH}$ adaptation and $\mathrm{Ca}^{2+}$ homeostasis. Furthermore, silencing of cpvmal resulted in significantly decreased accumulation of hypoviral RNA. Taken together, our results indicate that Cpvma1 plays an important role in the regulation of phenotypic traits and virulence and the accumulation of hypovirus RNA in C. parasitica.
\end{abstract}

The vacuolar $\mathrm{H}^{+}$-ATPases (V-ATPases) are a family of ATPdependent proton pumps localized to a variety of eukaryotic cellular membranes, including endosomes, lysosomes, Golgi-derived vesicles, secretory vesicles, and, in some cells, the plasma membrane. They function to acidify intracellular compartments and, in some cases, transport protons across the plasma membrane (Forgac 2007; Kane 2006). V-ATPases are large, complex enzymes composed of two distinct regions: the peripheral $\mathrm{V}_{1}$ domain and the integral $\mathrm{V}_{0}$ domain. $\mathrm{V}_{1}$ is composed of eight subunits (A to $\mathrm{H}$ ) and is responsible for ATP hydrolysis. The $\mathrm{V}_{0}$ domain, which carries out proton transport, is composed of six subunits (a, c, d, e, c', and c") (Jefferies et al. 2008). Intracellular V-ATPases play an important role in normal cellular processes such as receptor-mediated endocytosis, intracellular membrane trafficking, prohormone processing, protein degradation, and neurotransmitter uptake (Toei et al. 2010). VATPase-dependent acidification of the endosomal interior is crucial for the entry of various pathogenic agents and toxins (Cotter et al. 2015). V-ATPases are reported to facilitate the release of the influenza A virus genome into cytoplasm by acidifying the endosomal interior (Marjuki et al. 2011). In addition, plasma membrane V-ATPases function in $\mathrm{pH}$ homeostasis, bone resorption, and various disease processes (Jefferies et al. 2008).

Molecular genetic studies with Saccharomyces cerevisiae suggest that V-ATPase and vacuolar acidification influence a wide range of cellular activities. Disruption of genes encoding different subunits of V-ATPase in this organism was nonlethal. Although the mutant strains grew reasonably well in acidic medium $(\mathrm{pH} \mathrm{5.5),}$

†Corresponding author: B. Chen; chenyaoj@gxu.edu.cn

Funding: This work was supported by the National Basic Research Program of China (973 Program, grant 2015CB150600) and the National Natural Science Foundation of China (grants 31560043 and 31760498 to R. Li and 31170137 to B. Chen).

*The $e$-Xtra logo stands for "electronic extra" and indicates that six supplementary figures are published online.

The author(s) declare no conflict of interest.

(c) 2019 The American Phytopathological Society they failed to grow in alkaline medium (above $\mathrm{pH} 7$ ). They were also sensitive to a high concentration of $\mathrm{Ca}^{2+}$ in the medium and were defective in many vacuolar activities (Anraku et al. 1992; Hirata et al. 1990; Nelson and Nelson 1990). Similarly, a disruptant of the Aspergillus oryzae vmal gene, which encodes the catalytic subunit A of V-ATPase, showed abnormally shrunken vacuoles and hyphal walls at $\mathrm{pH} 8.5$ and a defect in growth at $\mathrm{pH} 10.0$. This indicates a role for vmal in alkaline $\mathrm{pH}$ adaptation and maintenance of proper vacuolar structure (Kuroki et al. 2002). Moreover, the vmal gene in Neurospora crassa was reported to be necessary for normal morphological development (Bowman et al. 2000). Disruption of the vmal gene results in inhibition of normal growth and abolished sporulation in Aspergillus nidulans. The vmal mutant was not viable at $\mathrm{pH}$ values above 7 and was highly sensitive to high $\mathrm{Zn}^{2+}$ concentrations (Melin et al. 2004). In contrast, deletion of the vmal gene in Ashbya gossypii did not cause a $\mathrm{pH}$-conditional lethal phenotype, although the mutant strain grew more slowly than the wild type and failed to form generative spores (Förster et al. 1999).

Cryphonectria parasitica is a filamentous fungus responsible for chestnut blight disease (Van Alfen et al. 1975). The fungus hosts a wide range of viruses and serves as a model filamentous fungus to study virus-host and virus-virus interactions (Eusebio-Cope et al. 2015). Infection by hypoviruses, a group of positive-sense RNA viruses, results in virulence attenuation (hypovirulence) to the fungus (Dawe and Nuss 2001). In addition, hypovirus infection also alters fungal phenotypic traits such as female infertility, reduced sporulation, pigmentation, laccase secretion, and oxalate accumulation (Nuss 2005). The molecular basis for these phenotypic changes involves alterations in host transcriptional profiles (Allen and Nuss 2004; Deng et al. 2007) as well as metabolic (Dawe et al. 2009) and proteomic (Wang et al. 2013, 2014) changes in response to hypovirus infection. In our previous comparative vesicle proteomics analysis, the expression level of Cpvma1 protein, a VATPase catalytic subunit A, was upregulated in hypovirus-infected C. parasitica strain EP155/CHV1-EP713. In accordance with the role of V-ATPase, it is likely that an elevated level of Cpvma1 in fungal vesicles may facilitate hypovirus transportation in and between fungal cells (Wang et al. 2013). To gain more insight into the role of the Cpvma1 protein in the virulence regulation of the chestnut blight fungus, we characterized Cpvma1 by constructing 
cpvmal-overexpressing and cpvmal-silenced strains. Our results demonstrate that Cpvma1 is required for the development of multiple traits, including virulence in $C$. parasitica, and it plays an important role in hypovirus RNA accumulation.

\section{MATERIALS AND METHODS}

Fungal strains, growth conditions, and transformation. The $C$. parasitica strains were the wild-type strain EP155 (ATCC

TABLE 1. Primers used in this study

\begin{tabular}{|c|c|c|}
\hline Primer $^{\mathrm{a}}$ & Sequence $\left(5^{\prime}-3^{\prime}\right)$ & Description \\
\hline OE-cpvmal-F & AAATATGCGGCCGCATGGCTCCGGTAAGCTAAGC & Construction of cpvmal-overexpressing vector \\
\hline OE-cpvmal-R & AAAGTTAACTTAATCGTCCATGACAGAGGC & \\
\hline pCPXHY $2-\mathrm{Pgpd}-\mathrm{F}$ & ACCTCCACTAGCTCCAGCCAAG & Verification of cpvmal-overexpressing stains \\
\hline pCPXHY 2 -Tgpd-R & AGGTGTTGGTCGCAGTCTAGGC & \\
\hline Cpvmal-probe-F & ACCAGATGCTATTGAGAGACG & Southern blot probe \\
\hline Cpvmal-probe-R & GGAGAAGGCTGAGGGAAATGA & \\
\hline RNAi-cpvmal (1)-F & GGCCGCAAGCTTAGGCAATGCTTCTCACCTGCTT & Construction of cpvmal-silenced vector \\
\hline RNAi-cpvmal (1)-R & TCTCCTTGACAGCCTTCGT & \\
\hline RNAi-cpvmal (2)-F & AGGCTGTCAAGGAGACTTGTACTCACGGTGGTCG & \\
\hline RNAi-cpvmal (2)-R & GAGCTCGGTACCCACCAATGCTTCTCACCTGCTT & \\
\hline RNAi-cpvmal (intron)-F & GTACTCTTACAAGAGCCACAATGCC & Verification of cpvmal-silenced stains \\
\hline RNAi-cpvmal (intron)-R & TGCAAAATATAACAAAAGCTGCCGAGAGGGTTGATT & \\
\hline pFCNH-Pgpd-F & АССТCCACTAGCTCCAGCCAAG & \\
\hline pFCNH-Tgpd-R & AGGTGTTGGTCGCAGTCTAGGC & \\
\hline RT-Cpvmal-F & TAAGAAGTTCCCTATTCCTATCC & qRT-PCR for cpvmal expression \\
\hline RT-Cpvmal-R & AACCTCTGCCATCTCGTTT & \\
\hline RT-18S-F & TCTCGAATCGCATGGCCT & \\
\hline RT-18S-R & TTACCCGTTGTAACCACGGC & \\
\hline RTQ1 & GTCGACGTAGGATCGTCTAC & qRT-PCR for viral RNA \\
\hline RTQ2 & TTGCGCAGGACGGTAACACT & \\
\hline
\end{tabular}

a $\mathrm{F}=$ forward, $\mathrm{R}=$ reverse, and $\mathrm{qRT}=$ quantitative reverse transcription.

A

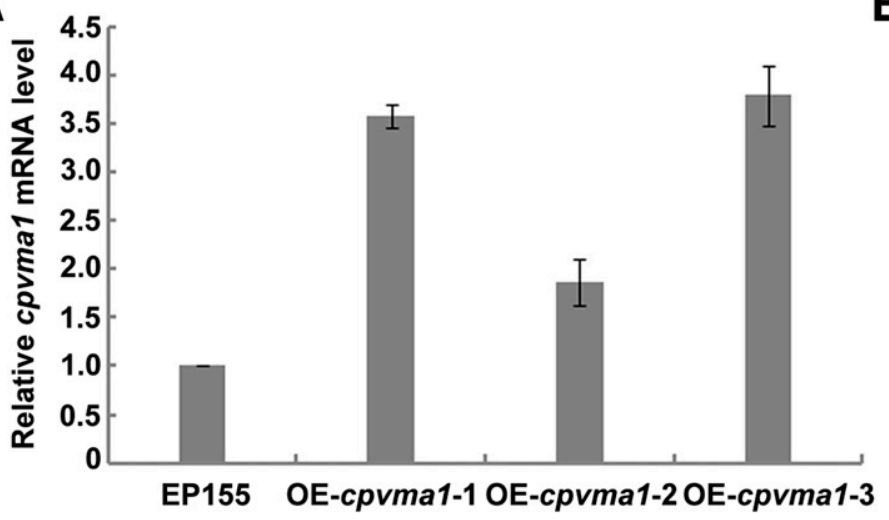

Strains

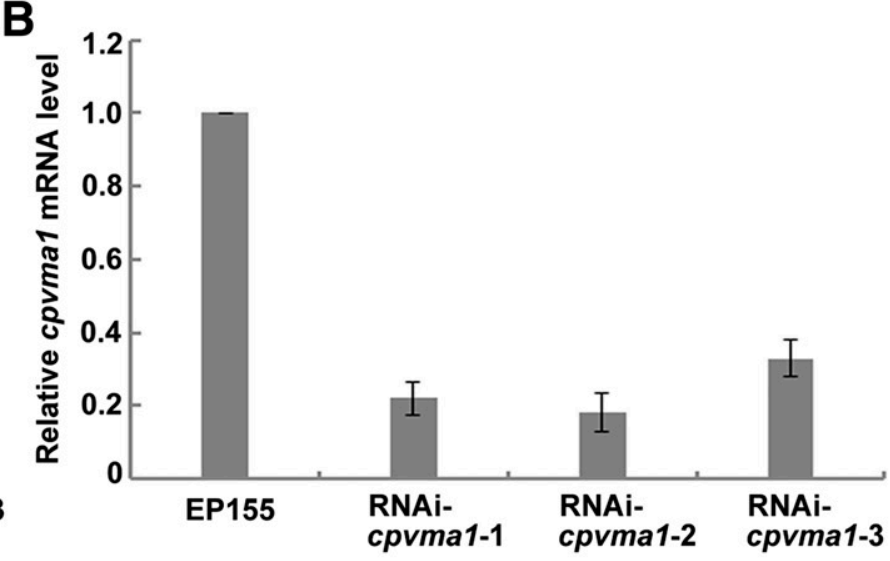

Strains

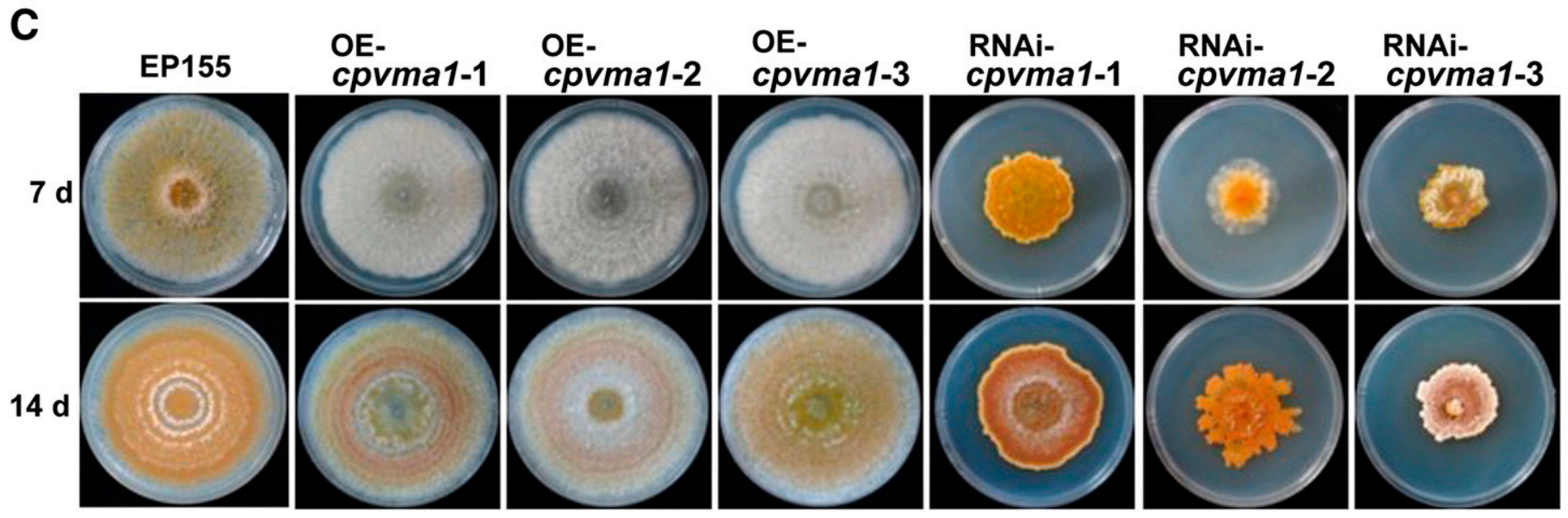

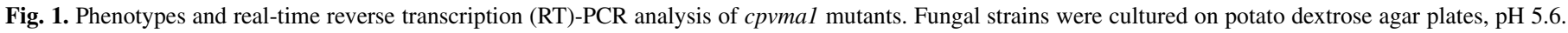

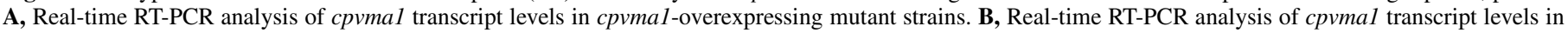

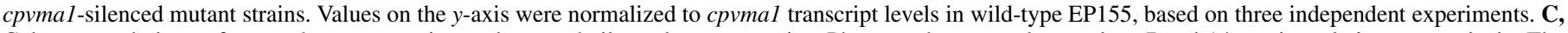

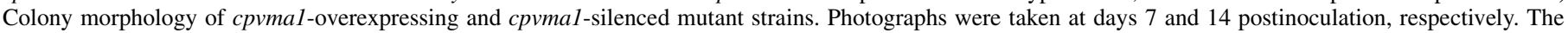

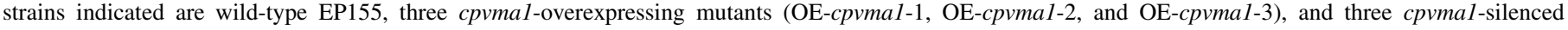
mutants (RNAi-cpvmal-1, RNAi-cpvmal-2, and RNAi-cpvmal-3). 
no. 38755) and its isogenic strain EP155/CHV1-EP713, which is transfected with a synthetic transcript of CHV1-EP713. The fungal strains were maintained on potato dextrose agar (PDA) at $26^{\circ} \mathrm{C}$ under a constant light/dark (12-h/12-h) cycle (Shapira et al. 1991). EP complete medium was used for liquid culture, and the strains were incubated at $26^{\circ} \mathrm{C}$ for 3 days with shaking at $100 \mathrm{rpm}$ (Puhalla and Anagnostakis 1971). Radial growth on plates was assessed by measuring the diameter of the colonies. The mycelium used for DNA, RNA, and protein extraction was collected and lyophilized as described previously (Powell and Van Alfen 1987). Preparation and transformation of $C$. parasitica strain EP155 spheroplasts was performed as described previously (Churchill et al. 1990; Shi et al. 2014). Transformants growing on the selective medium were chosen for further studies and single spored to ensure that each fungal transformant was derived from a single nucleus.

Plasmid construction. To overexpress cpvmal, a full-length cpvmal open reading frame (ORF) was amplified with the forward (F) and reverse (R) primers OE-cpvmal-F and OE-cpvmal-R, which incorporate NotI and HpaI sites, respectively; detailed primer sequences are listed in Table 1. The resulting 3.8-kb PCR amplicon was cloned into the Not $\mathrm{I} / \mathrm{HpaI}$-digested vector pCPXHY 2 under the control of the gpd promoter. The final expression construct was termed pCPXHY2-cpvmal (Supplementary Fig. S1). Each clone was sequenced to verify the accuracy of the sequence.

To knock down cpvmal, the cpvmal gene was amplified by PCR from $C$. parasitica genomic DNA with primers RNAi-cpvmal (1)-F and RNAi-cpvmal (1)-R and RNAi-cpvmal (2)-F and RNAi-cpvmal

TABLE 2. Effect of cpvmal mutations on sporulation ${ }^{\mathrm{a}}$

\begin{tabular}{lc}
\hline Fungal strain & Conidial spores/cm \\
\hline EP155 & $3.99 \times 10^{6} \pm 1.28 \times 10^{5}$ \\
OE-cpvmal-1 & $4.15 \times 10^{6} \pm 1.26 \times 10^{5}$ \\
OE-cpvmal-2 & $4.06 \times 10^{6} \pm 1.06 \times 10^{5}$ \\
OE-cpvmal-3 & $3.26 \times 10^{6} \pm 7.97 \times 10^{4}$ \\
RNAi-cpvmal-1 & $7.71 \times 10^{5} \pm 1.74 \times 10^{5 *}$ \\
RNAi-cpvmal-2 & $1.32 \times 10^{5} \pm 1.53 \times 10^{4 *}$ \\
RNAi-cpvmal-3 & $1.05 \times 10^{6} \pm 4.80 \times 10^{4 *}$ \\
\hline
\end{tabular}

a Strains were cultured on potato dextrose agar plates at $26^{\circ} \mathrm{C}$ for 14 days. Then the conidiation levels were measured on a colony area basis (in square centimeters). The mean and standard deviation were calculated from three replicates. An asterisk indicates a statistically significant difference from EP155 $(P \leq 0.05)$.

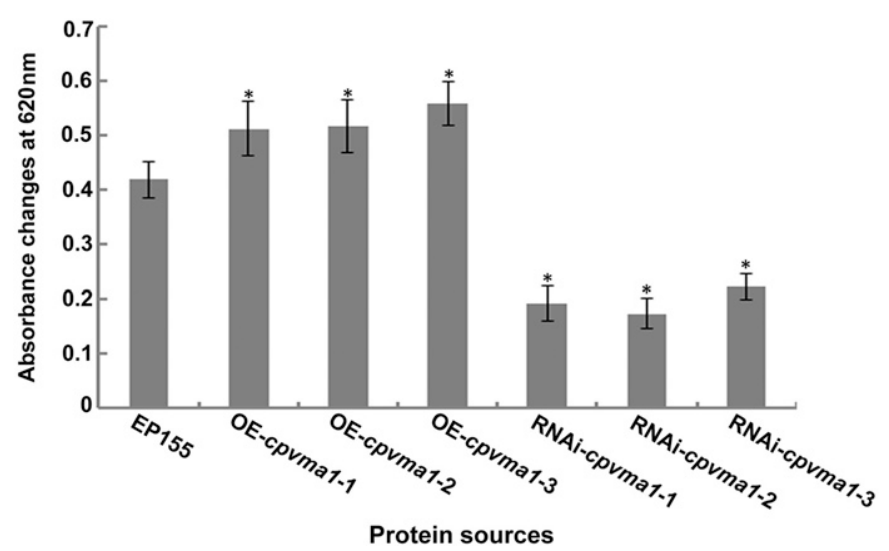

Fig. 2. Vacuolar $\mathrm{H}^{+}$-ATPase (V-ATPase) activity of cpvmal-overexpressing and cpvmal-silenced mutants. V-ATPase activity was measured using the ATPase Activity Assay Kit, in which enzymatic activity was indicated as the difference in absorption at $620 \mathrm{~nm}$ in the presence and absence of $1 \mu \mathrm{M}$ of Bafilomycin A1. The cpvmal-silenced mutants showed significantly reduced V-ATPase activity compared with wild-type strain EP155, whereas overexpressing cpvmal increased V-ATPase activity. The assays were performed in triplicate for each strain. An asterisk indicates a statistically significant difference from $\operatorname{EP} 155(P \leq 0.05 ; t$ test $)$.
(2)-R, respectively. The 738-bp amplicon, fragment RNAi-cpvmal (1) including the forward interference segment and intron 7 , and the 553-bp amplicon, fragment RNAi-cpvmal (2) including the reverse interference segment, were ligated into the $N o t \mathrm{I} / K p n \mathrm{I}$-digested vector pFCNH-intron using the In-Fusion HD Cloning Kit (Takara). The final construct was named pFCNH-cpvmal.

DNA extraction and Southern blot analysis. Genomic DNA was extracted from $C$. parasitica using the method described by Churchill et al. (1990). Southern blot analysis was performed as described previously (Sambrook and Russell 2001). After digestion with restriction enzyme SalI, the DNA was transferred to a nylon membrane after separation in a $1 \times$ TBE, $0.8 \%$ agarose gel.

Measurement of V-ATPase activity. The Pi generated by VATPase activity was determined as the molybdate complex by optical absorbance measured at $620 \mathrm{~nm}$ using the ATPase Activity Assay Kit (catalog no. MAK113; Sigma-Aldrich). Reaction
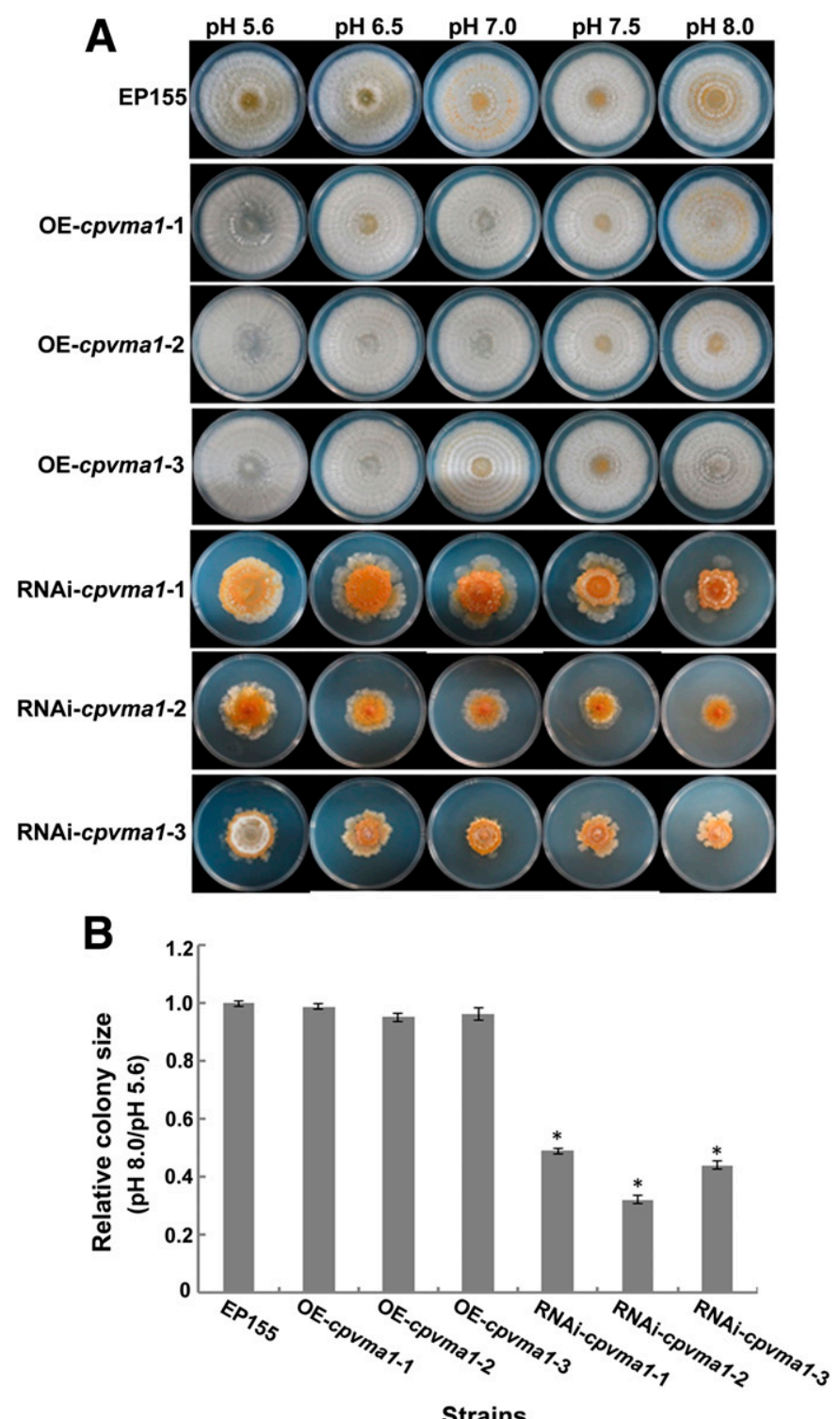

Fig. 3. Growth properties of cpvmal-overexpressing and cpvmal-silenced mutants on potato dextrose agar plates adjusted to $\mathrm{pH} 5.6$ to 8.0. A, Colony morphology of cpvmal-overexpressing and cpvmal-silenced mutants. Colonies after 7 days of culture are shown. B, Relative colony size of cpvmaloverexpressing and cpvmal-silenced mutants. The relative colony size of the indicated strain under $\mathrm{pH} 8.0$ to $\mathrm{pH} 5.6$ is shown on the $y$-axis. The assays were repeated three times. The error bars represent standard deviations. An asterisk indicates a statistically significant difference from EP155 $(P \leq 0.05 ; t$ test). 
mixtures were preincubated for 10 min with or without $1 \mu \mathrm{M}$ of Bafilomycin A1 (catalog no. B1793; Sigma-Aldrich) and reactions were initiated by the addition of $4 \mathrm{mM}$ of ATP. V-ATPase activity was defined operationally as the difference in the rate of ATP hydrolysis in the presence and absence of $1 \mu \mathrm{M}$ of Bafilomycin A1. Total protein extracts were prepared as described (Shi et al. 2014). Enzymatic assays were performed using total protein extracts in triplicate. Significance analysis was performed with the $t$ test.

Virulence assay. Dormant stems of Chinese chestnut (Castanea mollissima) were used for the virulence assay according to Shi et al. (2014). The stems were incubated in a plastic bag at $26^{\circ} \mathrm{C}$ to allow lesion development. Canker sizes were analyzed at 25 days postinoculation. The infection assays were repeated three times for each fungal strain.

Transmission of the hypovirus. Virus transmission by anastomosis was performed as described previously (Cortesi et al. 2001). Briefly, mycelial plugs of the virus-containing strain

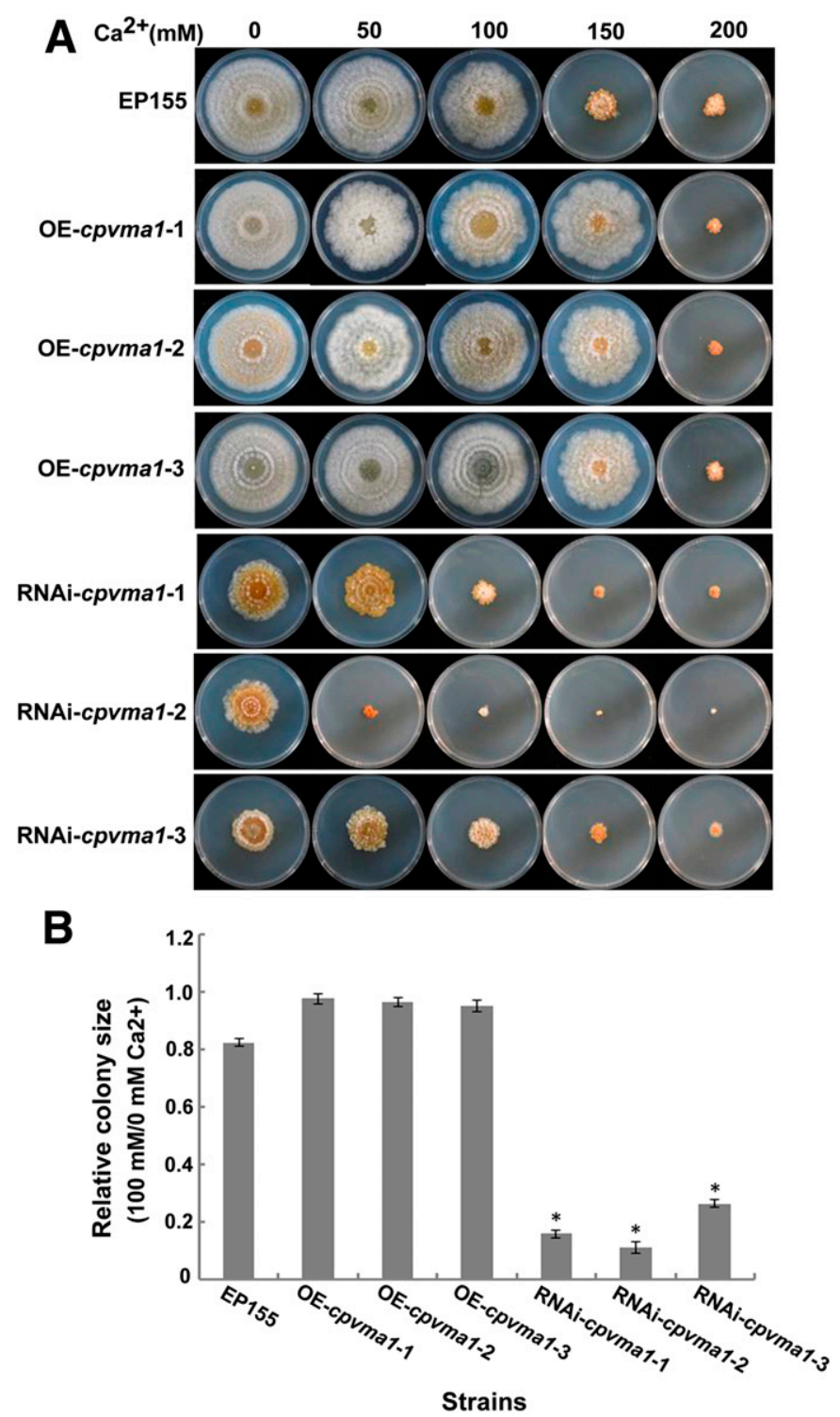

Fig. 4. Growth properties of cpvmal-overexpressing and cpvmal-silenced mutants on potato dextrose agar plates supplemented with $\mathrm{CaCl} 2$. $\mathbf{A}$, Colony morphology of cpvmal-overexpressing and cpvmal-silenced mutants. Colonies after 7 days of culture are shown. B, Relative colony size of cpvmaloverexpressing and cpvmal-silenced mutants. The relative colony size of the indicated strain at 100 to $0 \mathrm{mM} \mathrm{Ca}^{2+}$ is shown on the $y$-axis. The assays were repeated three times. The error bars represent standard deviations. An asterisk indicates a statistically significant difference from EP155 $(P \leq 0.05 ; t$ test $)$.
EP155/CHV1-EP713 were cocultured with adjacent mycelial plugs of virus-free recipient transformants with hygromycin-resistance marker on PDA medium. After 5 days of coculture, putatively fused mycelia at the border between each pair of strains were transferred to hygromycincontaining PDA plates and examined for the occurrence of a sector showing different phenotypes. Mycelia in the sector were transferred to fresh hygromycin-containing PDA plates and were single spored to find virus-infected recipient transformants. The presence of hypovirus was confirmed by isolation of dsRNA from single-spored strains.

RNA extraction and gene transcript quantification. Fungal RNA was extracted as described previously (Allen et al. 2003). Total nucleic acids were extracted sequentially with phenol, phenol-chloroform, and chloroform-isoamyl alcohol. The nucleic acid samples were exposed to RQ1-DNase (Promega) digestion for $2 \mathrm{~h}$ at $37^{\circ} \mathrm{C}$. Electrophoresis in a $0.8 \%$ agarose gel was used to analyze the RNA quality and quantity.

The mRNA expression of cpvmal in the transformed strains was examined with quantitative real-time reverse transcription (RT)PCR. Total fungal RNA was reverse-transcribed into cDNA using the RevertAid First Strand cDNA Synthesis Kit (Fermentas). 18S rRNA served as the normalization reference. The relative quantification of cpvmal mRNA expression was evaluated by the comparative cycle threshold $(-\Delta \Delta \mathrm{Ct})$ as described previously (Shi et al. 2014). Primer sequences used are listed in Table 1.

Viral dsRNA semiquantification. A quantity of $5 \mu \mathrm{g}$ of RNA was separated in a $0.8 \%$ agarose gel and analyzed by densitometry with the aid of Quantity One gel analysis software to quantify the relative amount of dsRNA in each sample. The florescence intensities of the viral dsRNA bands were normalized against the $18 \mathrm{~S}$ rRNA in the samples.

Viral ssRNA quantification. The relative accumulation levels of viral ssRNA were determined by quantitative real-time PCR as described previously by Lin et al. (2007). cDNAs specific for viral plus- and minus-strand RNA were generated with primers RTQ2 and RTQ1, respectively, using a RevertAid First Strand cDNA Synthesis Kit. Each cDNA synthesis reaction mixture contained primer RT-18S-R, which was complementary to the portion of $C$. parasitica $18 \mathrm{~S}$ rRNA. The primer sets used for realtime PCR were RTQ1 and RTQ2 for the viral RNA and RT-18S-R/F for $18 \mathrm{~S}$ rRNA. Viral cDNA values were normalized against the cDNA value of $18 \mathrm{~S}$ rRNA.

\section{RESULTS}

Characterization of the cpvmal gene from $C$. parasitica. Nucleotide sequence analysis of cpvmal genomic DNA and cDNA revealed an ORF of 3,869 bp. This ORF is located on scaffold 10 of the $C$. parasitica EP155 genome (https://genome.jgi.doe.gov/ Crypa2/Crypa2.home.html) (Eusebio-Cope et al. 2015), includes seven introns, encodes a putative protein product of 1,059 amino acids, and has a predicted molecular mass of $116.7 \mathrm{kDa}$ (Supplementary Fig. S2A). Analysis of the deduced Cpvma1 amino acid sequence revealed the presence of three conserved domains: an $\mathrm{N}$-terminal NtpA domain between amino acids 12 and 274 that is related to an archaeal/vacuolar-type $\mathrm{H}^{+}$-ATPase catalytic subunit A/ Vma1, a Hom-end-hint domain between amino acids 275 and 722 , and the C-terminal NtpA domain between amino acids 723 and 1,055. A homology search using the deduced amino acid sequence showed that the Cpvma1 protein is related to fungal Vma1 proteins from N. crassa (86\%), Sporothrix insectorum (78\%), Fonsecaea monophora (64\%), Candida maltosa $(62 \%)$, and S. cerevisiae $(59 \%)$. Southern blot analysis showed that there is only a single copy of this gene in the $C$. parasitica genome (Supplementary Fig. S3C).

Phenotypes of the cpvmal-overexpressing and cpvmalsilenced strains. Cpvmal-overexpressing transformants were first selected on PDA plates supplemented with hygromycin. After initial screening by PCR, single-spore-derived transformants were further confirmed to carry the introduced construct fragment by 
Southern blot analysis. Confirmed transformants were designated as OE-cpvmal strains and two to three copies of cpvmal were introduced into the transformants. Quantitative RT-PCR showed that cpvmal transcript levels in the transformants were 1.9 to 3.8 times as high as wild-type strain EP155 (Fig. 1A). The transformants accumulated apparently less orange pigment compared with the wild-type strain (Fig. 1C). No significant change in conidial sporulation was observed in the cpvmal-overexpressing strains determined at day 14 (Table 2).

Because attempts to disrupt the cpvmal gene by homologous recombination were unsuccessful, we opted to suppress cpvmal expression by using an RNAi strategy. The single-spore-derived isolates were confirmed to harbor the silencing fragment by PCR (Supplementary Fig. S4). Confirmed transformants were designated as RNAi-cpvmal strains. RT-PCR quantification revealed that the three selected cpvmal-silenced strains expressed a much lower level (about 18 to 33\%) of cpvmal transcript than wild-type strain EP155 (Fig. 1B). The cpvmal-silenced strains grew much more slowly on PDA plates and exhibited both enhanced pigment and distinct colony morphology (Fig. 1C). The numbers of conidia per colony area produced by the cpvmal-silenced strains were significantly lower than those of EP155 (Table 2).

Enzymatic characterization of cpvmal strains. Compared with wild-type EP155, the cpvmal-overexpressing strains exhibited an increase of 25 to $38 \%$ in V-ATPase activity. In contrast, cpvmal-silenced strains recorded a decrease of 47 to $60 \%$ in VATPase activity (Fig. 2). These results established a positive correlation between cpvmal expression and V-ATPase activity in this fungus.

Response of cpvmal strains to elevated $\mathrm{pH}$ and $\mathrm{Ca}^{2+}$. Previous reports showed that the yeast VMA deletion mutants failed to grow in basic medium (above $\mathrm{pH} 7$ ) and were sensitive to high concentrations of $\mathrm{Ca}^{2+}$ (Nelson and Nelson 1990). On PDA medium, the wild-type and cpvmal-overexpressing strains grew well at a pH range from 5.6 to 8.0, but the cpvmal-silenced strains were inhibited at all pH levels, especially at pH 8.0 (Fig. 3). High $\mathrm{Ca}^{2+}(100 \mathrm{mM})$ inhibited the growth of cpvmal-silenced strains, whereas the cpvmal-overexpressing strains grew as well as or even better than the wild type at all tested $\mathrm{Ca}^{2+}$ levels (Fig. 4).

Cpvmal is essential for virulence in $\boldsymbol{C}$. parasitica. The cpvmal strains were inoculated onto dormant stems of Chinese chestnut to test whether overexpressing or silencing of the cpvmal gene affected virulence. The cpvmal-overexpressing strains showed similar virulence with the wild-type strain EP155, whereas the cpvmal-silenced strains incited significantly smaller cankers (Fig. 5), demonstrating that cpvmal is essential for virulence in C. parasitica.

Accumulation of hypovirus RNA in cpvmal-overexpressing and cpvmal-silenced strains. To examine the effect of cpvmal on hypovirus infection, we introduced hypovirus CHV1EP713 into the cpvmal strains via anastomosis by paring the cpvmal strains with hypovirus-containing isogenic strain EP155/ CHV1-EP713. Hypovirus-infected cpvmal strains exhibited a regular hypovirus infection phenotype that included a lower growth rate, reduced pigmentation, and a lower level of sporulation. Of particular note was that hypovirus infection severely inhibited the growth of cpvmal-overexpressing strains (Fig. 6A). Agarose gel analysis of viral dsRNA isolated from the hypovirus-infected cpvmal-overexpressing strains revealed that the accumulation level of CHV1-EP713 viral RNA was comparable with that observed in strain EP155/CHV1-EP713. In contrast, hypovirus-infected cpvmal-silenced strains seemed to accumulate a much lower level of viral RNA (Fig. 6B). Quantification of viral RNA yielded similar results (Fig. 6B and C; Supplementary Fig. S5). These results suggested that the efficient replication or accumulation of CHV1EP713 requires a normal level of cpvmal.

A

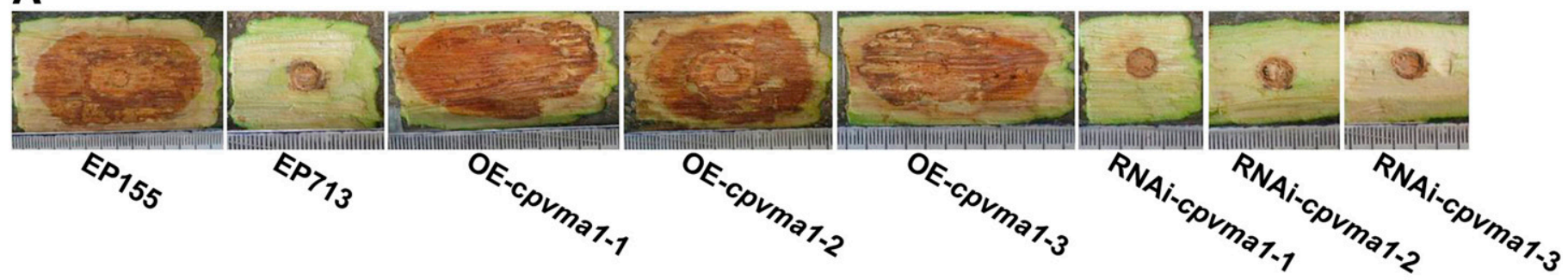

B

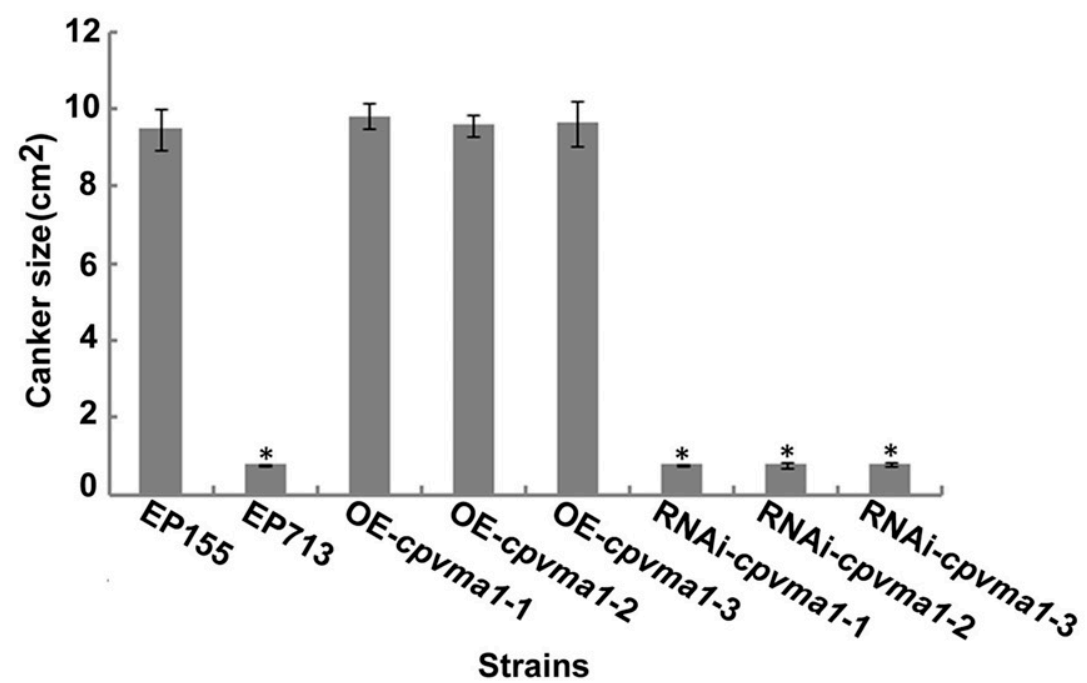

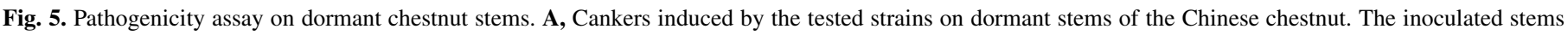

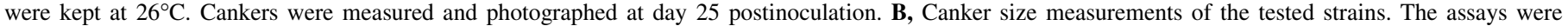
performed in triplicate for each strain. An asterisk indicates a statistically significant difference from EP155 ( $P \leq 0.05 ; t$ test). 


\section{DISCUSSION}

Prior studies reported that V-ATPase is likely indispensable for cell viability in some organisms. For instance, deletion of the gene encoding the V-ATPase subunit B in Drosophila melanogaster resulted in a larval lethal phenotype (Davies et al. 1996). In Dictyostelium discoideum, attempts to disrupt the gene encoding the proteolipid C subunit of the V-ATPase were unsuccessful (Xie et al. 1996). Similarly, we failed to obtain cpvmal knockout strains by homologous recombination in $C$. parasitica (data not shown).
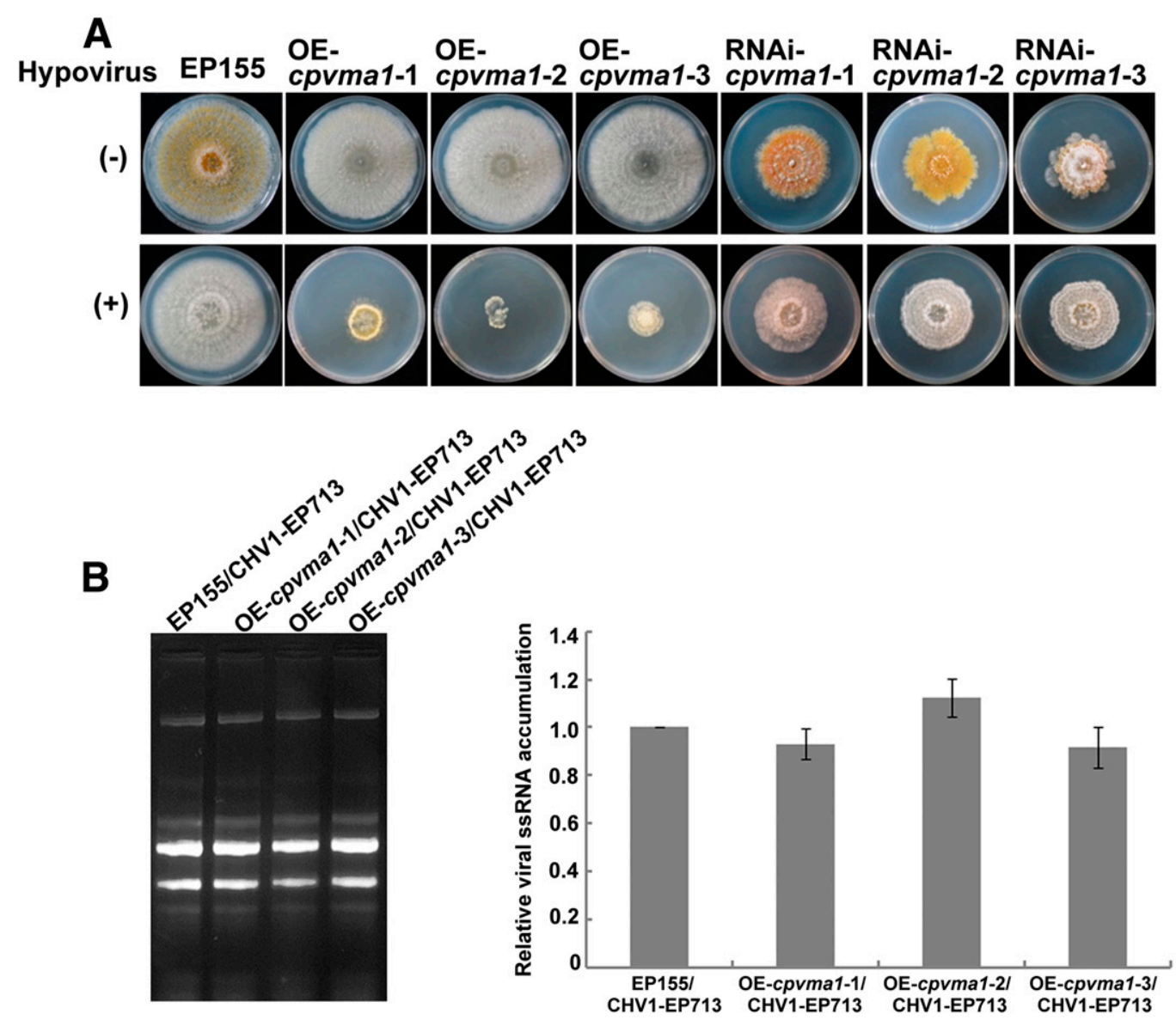

Strains

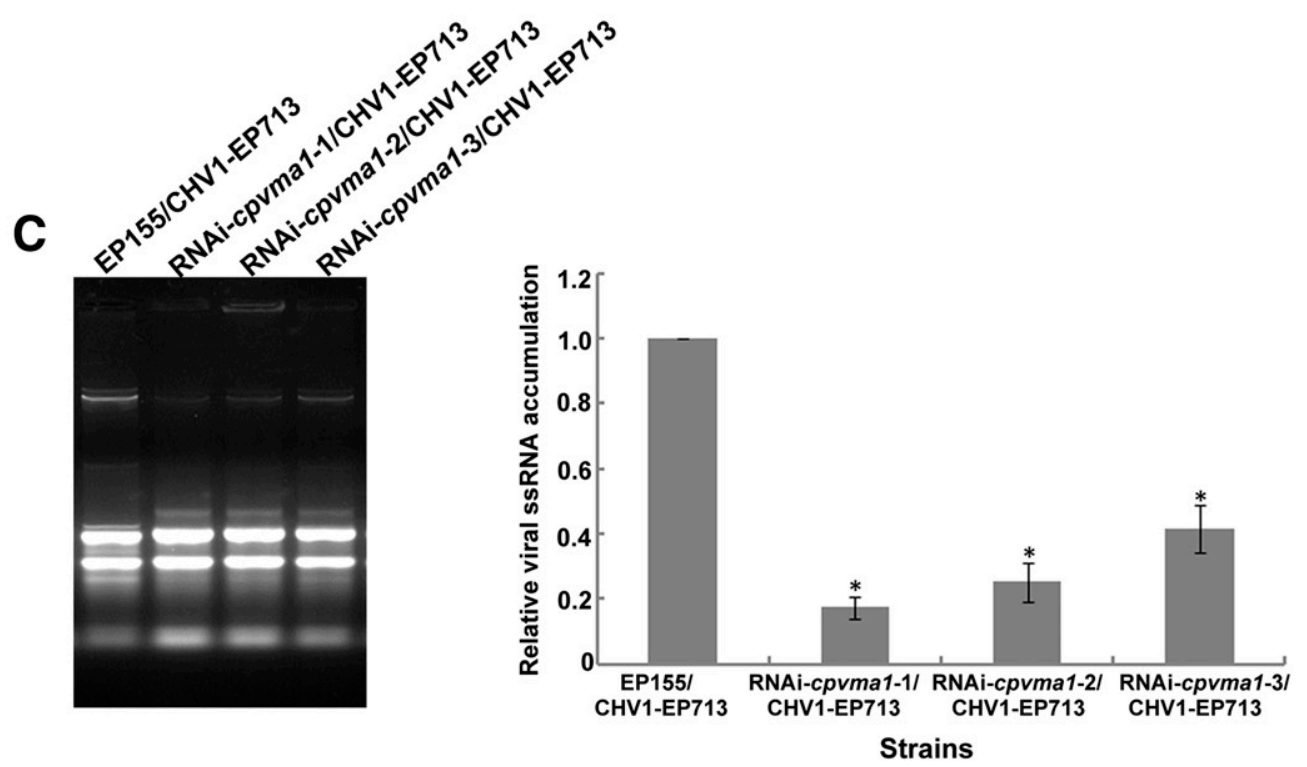

Fig. 6. Colony morphology and viral RNA accumulation in hypovirus-containing fungal hosts. A, Colony morphology of hypovirus-free and hypovirus-containing host fungal strains on potato dextrose agar. The photograph was taken at day 7 postinoculation. B, Agarose gel electrophoresis (left) and reverse transcription (RT)PCR quantification (right) of viral ssRNA accumulation from hypovirus-containing cpvmal-overexpressing transformants. C, Agarose gel electrophoresis (left) and RT-PCR quantification (right) of viral ssRNA accumulation from hypovirus-containing cpvmal-silenced transformants. The viral ssRNA level of EP155/ CHV1-EP713 was set at 1.0, and the levels of indicated hypovirus-containing strains were expressed as the fold change relative to those in EP155/CHV1-EP713. Values were calculated from three independent experiments, with an asterisk indicating statistical significance relative to EP155/CHV1-EP713 $(P \leq 0.05 ; t$ test). 
Based on these results, we suspected that cpvmal might be an essential gene in C. parasitica. Alternatively, we opted to employ an RNAi strategy to knock down cpvmal in the cells. This approach was successfully used in $C$. parasitica previously (Gullusci and Turina 2007; Jacob-Wilk et al. 2009; Rostagno et al. 2010). In contrast, V-ATPase genes are clearly not essential in the yeast $S$. cerevisiae and the closely related filamentous fungus Ashbya gossypii, in which deletion strains were characterized in considerable detail (Förster et al. 1999; Hirata et al. 1990). Moreover, deletion of V-ATPase genes was not lethal but did cause a number of growth defects in the filamentous fungus N. crassa, Aspergillus nidulans, and Beauveria bassiana (Bowman et al. 2000; Melin et al. 2004; Zhu et al. 2017). These differences may explain why the biological function of the corresponding genes can be varied in different fungal species although V-ATPase components may be phylogenetically conserved.

Previous studies demonstrated that loss of function of the VATPase subunit genes in some fungi results in a growth defect, abnormal vacuole morphology, reduced asexual spore production, and attenuated virulence (Bowman et al. 2000; Förster et al. 1999; Hirata et al. 1990; Melin et al. 2004; Zhu et al. 2017). As reported in this work, the cpvmal-silenced strains exhibited a phenotype of reduced growth rate, lower level of sporulation, and decreased virulence. Nevertheless, because overexpression of the cpvmal gene did not show any changes in fungal virulence and severe growth retardation of the cpvmal-silenced strains was observed, changes in virulence of the engineered strains may simply result from the growth defect. Therefore, if virulence determinants are defined as factors that affect only virulence, cpvmal may not be considered as a specific virulence factor, but rather an important gene for normal fungal growth and development. Moreover, the finding that silencing cpvmal significantly reduced the total VATPase activity of $C$. parasitica further validated the assumption that Cpvma1 is the major component of the intracellular V-ATPase.

The cpvmal-silenced strains showed greater $\mathrm{pH}$ sensitivity, growing well at $\mathrm{pH} 5.6$ but inhibited at $\mathrm{pH}$ 8.0. The cpvmaloverexpressing strains grew well in the range of $\mathrm{pH} 5.6$ through 8.0 (Fig. 3). Our results somewhat contradict those from previous studies of $S$. cerevisiae in which the V-ATPase deletion strains failed to grow in alkaline medium above pH 7 (Nelson and Nelson 1990). This discrepancy may be attributable to the fact that silencing might have only a quantitative effect on reducing the amount of functional protein, and the remaining enzyme could still execute its physiological functions to some extent.

V-ATPase activity is essential for a microbe to adapt to high $\mathrm{Ca}^{2+}$ environment. In $S$. cerevisiae, high $\mathrm{Ca}^{2+}$ concentrations strongly inhibit growth of $V m a$ disruptant strains (Ohya et al. 1991). Similarly, high concentrations $(100 \mathrm{mM})$ of $\mathrm{Ca}^{2+}$ in the medium restricted the growth of the cpvmal-silenced strains by approximately 75 to $90 \%$, whereas the wild-type strain was affected to a lesser degree (inhibition of approximately 17\%; Fig. 4). This result indicates that Cpvma1 plays an important role in maintaining $\mathrm{Ca}^{2+}$ homeostasis.

V-ATPase-dependent acidification of the endosome was shown to be crucial for the entry of various enveloped viruses (Gruenberg and Van Der Goot 2006). Moreover, V-ATPase in Sindbis virus was shown to be required for production of viral components and postentry events in the host cell (Hunt et al. 2011). The cpvmaloverexpressing strains showed enhanced susceptibility to hypovirus infection compared with wild-type EP155, as the CHV1-EP713infected cpvmal-overexpressing strains became severely debilitated (Fig. 6A). Although a previous study showed that the Cpvma1 protein was upregulated by 2.9-fold in EP155/CHV1-EP713 (Wang et al. 2013), the effect of hypovirus infection on cpvmal transcript levels had not been determined. Accordingly, cpvmal expression in the virus-infected cpvmal-overexpressing strain and the wild-type strain were compared in this study. As shown in Supplementary Figure S6, the cpvmal transcript level in EP155 increased about 1.6-fold following CHV1-EP713 infection, whereas the cpvmal transcript level in the cpvmal-overexpressing/CHV1-EP713 was 3.3 to 4.5 times higher than that in EP155/CHV1-EP713, implying that the increase in cpvmal expression level may be beneficial to hypoviral replication.

Unexpectedly, viral RNA levels did not significantly accumulate in CHV1-EP713-infected cpvmal-overexpressing strains relative to the control strain EP155/CHV1-EP713 (Fig. 6B). A similar result was observed for $C$. parasitica dicer-like gene $d c l-2$ disruption mutants, in which no significant increase in hypovirus RNA accumulation was observed in infected $\Delta d c l-2$ cultures even though the infected fungi showed severe debilitation in response to CHV1EP713 infection (Segers et al. 2007). However, an increased viral RNA level was observed in the $\Delta d c l-2$ strain for a mutant CHV1EP713 that lacked the suppressor of RNA silencing p29, which accumulated a much lower level of viral RNA in the wild-type EP155 (Chiba and Suzuki 2015; Segers et al. 2007). Considering that hypovirus RNA accumulation decreased significantly in cpvmal-silenced strains (Fig. 6C), we assume that a normal level of cpvmal is required for efficient replication or accumulation of CHV1-EP713. Since vesicles are implicated to be the place where the virus replicates (Fahima et al. 1994; Hansen et al. 1985), we propose that Cpvma1 likely regulates hypovirus replication through affecting the acidification of vesicles. Thus, elucidation of the mechanism of Cpvma1 in the regulation of vesicle $\mathrm{pH}$ could be a key to understanding the cell environment required for hypovirus replication.

Genes encoding signal transduction pathway components (Choi et al. 1995, 2005; Deng et al. 2007; Kim et al. 2002; Moretti et al. 2014; Park et al. 2012; So et al. 2017) or metabolic pathways (Yao et al. 2013) are reported to be involved in conidiation, vegetative growth, and virulence in $C$. parasitica. However, the cpvmalsilenced phenotypes of hypovirulence, sensitivity to alkaline $\mathrm{pH}$, higher $\mathrm{Ca}^{2+}$ concentration, and significant reduction in the accumulation of hypovirus RNA are unusual. Similar observations were made previously in spontaneous $C$. parasitica mutant NB58F (Polashock et al. 1994) and in a transcription factor prol disruption mutant (Sun et al. 2009). The NB58F mutant was deficient in hypovirus maintenance. $\Delta$ prol was deficient in conidial production and the hypovirus-transferred $\Delta$ prol continually produced virusfree sectors, suggesting that prol is required for stable maintenance of hypovirus replication. Thus it would be worthwhile to investigate the relation between prol and cpvmal, since this may help to uncover the microenvironment required for hypovirus replication and maintenance.

\section{LITERATURE CITED}

Allen, T. D., Dawe, A. L., and Nuss, D. L. 2003. Use of cDNA microarrays to monitor transcriptional responses of the chestnut blight fungus Cryphonectria parasitica to infection by virulence-attenuating hypoviruses. Eukaryot. Cell 2:1253-1265.

Allen, T. D., and Nuss, D. L. 2004. Specific and common alterations in host gene transcript accumulation following infection of the chestnut blight fungus by mild and severe hypoviruses. J. Virol. 78:4145-4155.

Anraku, Y., Hirata, R., Wada, Y., and Ohya, Y. 1992. Molecular genetics of the yeast vacuolar H (+)-ATPase. J. Exp. Biol. 172:67-81.

Bowman, E. J., Kendle, R., and Bowman, B. J. 2000. Disruption of vma-1, the gene encoding the catalytic subunit of the vacuolar H (+)-ATPase, causes severe morphological changes in Neurospora crassa. J. Biol. Chem. 275: 167-176.

Chiba, S., and Suzuki, N. 2015. Highly activated RNA silencing via strong induction of dicer by one virus can interfere with the replication of an unrelated virus. Proc. Natl. Acad. Sci. USA 112: E4911-E4918.

Choi, E. S., Chung, H. J., Kim, M. J., Park, S. M., Cha, B. J., Yang, M. S., and Kim, D. H. 2005. Characterization of the ERK homologue CpMK2 from the chestnut blight fungus Cryphonectria parasitica. Microbiology 151: 1349-1358.

Choi, G. H., Chen, B., and Nuss, D. L. 1995. Virus-mediated or transgenic suppression of a G-protein alpha subunit and attenuation of fungal virulence. Proc. Natl. Acad. Sci. USA 92:305-309. 
Churchill, A., Ciuffetti, L., Hansen, D., Van Etten, H., and Van Alfen, N. 1990. Transformation of the fungal pathogen Cryphonectria parasitica with a variety of heterologous plasmids. Curr. Genet. 17:25-31.

Cortesi, P., McCulloch, C. E., Song, H., Lin, H., and Milgroom, M. G. 2001. Genetic control of horizontal virus transmission in the chestnut blight fungus, Cryphonectrica parasitica. Genetics 159:107-118.

Cotter, K., Stransky, L., McGuire, C., and Forgac, M. 2015. Recent insights into the structure, regulation, and function of the V-ATPases. Trends Biochem. Sci. 40:611-622.

Davies, S. A., Goodwin, S. F., Kelly, D. C., Wang, Z., Sözen, M. A., Kaiser, K., and Dow, J. A. 1996. Analysis and inactivation of vha55, the gene encoding the vacuolar ATPase B-subunit in Drosophila melanogaster reveals a larval lethal phenotype. J. Biol. Chem. 271:30677-30684.

Dawe, A. L., and Nuss, D. L. 2001. Hypoviruses and chestnut blight: Exploiting viruses to understand and modulate fungal pathogenesis. Annu. Rev. Genet. 35:1-29.

Dawe, A. L., Van Voorhies, W. A., Lau, T. A., Ulanov, A. V., and Li, Z. 2009. Major impacts on the primary metabolism of the plant pathogen Cryphonectria parasitica by the virulence-attenuating virus CHV1-EP713. Microbiology 155:3913-3921.

Deng, F., Allen, T. D., Hillman, B. I., and Nuss, D. L. 2007. Comparative analysis of alterations in host phenotype and transcript accumulation following hypovirus and mycoreovirus infections of the chestnut blight fungus Cryphonectria parasitica. Eukaryot. Cell 6:1286-1298.

Eusebio-Cope, A., Sun, L., Tanaka, T., Chiba, S., Kasahara, S., and Suzuki, N. 2015. The chestnut blight fungus for studies on virus/host and virus/virus interactions: From a natural to a model host. Virology 477:164-175.

Fahima, T., Wu, Y., Zhang, L., and Van Alfen, N. K. 1994. Identification of the putative RNA polymerase of Cryphonectria hypovirus in a solubilized replication complex. J. Virol. 68:6116-6119.

Forgac, M. 2007. Vacuolar ATPases: Rotary proton pumps in physiology and pathophysiology. Nat. Rev. Mol. Cell Biol. 8:917-929.

Förster, C., Santos, M. A., Ruffert, S., Krämer, R., and Revuelta, J. L. 1999. Physiological consequence of disruption of the VMA1 gene in the riboflavin overproducer Ashbya gossypii. J. Biol. Chem. 274:9442-9448.

Gruenberg, J., and Van Der Goot, F. G. 2006. Mechanisms of pathogen entry through the endosomal compartments. Nat. Rev. Mol. Cell Biol. 7:495-504.

Gullusci, M., and Turina, M. 2007. Silencing of cryparin, a cell wall hydrophobin, in Cryphonectria parasitica. J. Plant Pathol. 89:141-147.

Hansen, D. R., Van Alfen, N. K., Gillies, K., and Powell, W. A. 1985. Naked dsRNA associated with hypovirulence of Endothia parasitica is packaged in fungal vesicles. J. Gen. Virol. 66:2605-2614.

Hirata, R., Ohsumk, Y., Nakano, A., Kawasaki, H., Suzuki, K., and Anraku, Y. 1990. Molecular structure of a gene, VMA1, encoding the catalytic subunit of $\mathrm{H}(+)$-translocating adenosine triphosphatase from vacuolar membranes of Saccharomyces cerevisiae. J. Biol. Chem. 265:6726-6733.

Hunt, S. R., Hernandez, R., and Brown, D. T. 2011. Role of the vacuolarATPase in Sindbis virus infection. J. Virol. 85:1257-1266.

Jacob-Wilk, D., Turina, M., Kazmierczak, P., and Van Alfen, N. K. 2009. Silencing of Kex2 significantly diminishes the virulence of Cryphonectria parasitica. Mol. Plant-Microbe Interact. 22:211-221.

Jefferies, K. C., Cipriano, D. J., and Forgac, M. 2008. Function, structure and regulation of the vacuolar $(\mathrm{H}+)$-ATPases. Arch. Biochem. Biophys. 476: $33-42$.

Kane, P. M. 2006. The where, when, and how of organelle acidification by the yeast vacuolar H+-ATPase. Microbiol. Mol. Biol. Rev. 70:177-191.

Kim, M. J., Choi, J. W., Park, S. M., Cha, B. J., Yang, M. S., and Kim, D. H. 2002. Characterization of a fungal protein kinase from Cryphonectria parasitica and its transcriptional upregulation by hypovirus. Mol. Microbiol. 45:933-941.

Kuroki, Y., Juvvadi, P. R., Arioka, M., Nakajima, H., and Kitamoto, K. 2002. Cloning and characterization of vmaA, the gene encoding a 69-kDa catalytic subunit of the vacuolar H+-ATPase during alkaline $\mathrm{pH}$ mediated growth of Aspergillus oryzae. FEMS Microbiol. Lett. 209:277-282.

Lin, H., Lan, X., Liao, H., Parsley, T. B., Nuss, D. L., and Chen, B. 2007. Genome sequence, full-length infectious cDNA clone, and mapping of viral double-stranded RNA accumulation determinant of hypovirus CHV1EP721. J. Virol. 81:1813-1820.

Marjuki, H., Gornitzky, A., Marathe, B. M., Ilyushina, N. A., Aldridge, J. R., Desai, G., Webby, R. J., and Webster, R. G. 2011. Influenza A virus-induced early activation of ERK and PI3K mediates V-ATPase-dependent intracellular $\mathrm{pH}$ change required for fusion. Cell. Microbiol. 13:587-601.
Melin, P., Schnürer, J., and Wagner, E. G. 2004. Disruption of the gene encoding the V-ATPase subunit A results in inhibition of normal growth and abolished sporulation in Aspergillus nidulans. Microbiology 150:743-748.

Moretti, M., Rossi, M., Ciuffo, M., and Turina, M. 2014. Functional characterization of the three mitogen-activated protein kinase kinases (MAP2Ks) present in the Cryphonectria parasitica genome reveals the necessity of Cpkk1 and Cpkk2, but not Cpkk3, for pathogenesis on chestnut (Castanea spp.). Mol. Plant Pathol. 15:500-512.

Nelson, H., and Nelson, N. 1990. Disruption of genes encoding subunits of yeast vacuolar $\mathrm{H}(+)$-ATPase causes conditional lethality. Proc. Natl. Acad. Sci. USA 87:3503-3507.

Nuss, D. L. 2005. Hypovirulence: Mycoviruses at the fungal-plant interface. Nat. Rev. Microbiol. 3:632-642.

Ohya, Y., Umemoto, N., Tanida, I., Ohta, A., Iida, H., and Anraku, Y. 1991. Calcium-sensitive cls mutants of Saccharomyces cerevisiae showing a Petphenotype are ascribable to defects of vacuolar membrane $\mathrm{H}(+)$-ATPase activity. J. Biol. Chem. 266:13971-13977.

Park, J. A., Kim, J. M., Park, S. M., and Kim, D. H. 2012. Characterization of CpSte11, a MAPKKK gene of Cryphonectria parasitica, and initial evidence of its involvement in the pheromone response pathway. Mol. Plant Pathol. 13:240-250.

Polashock, J. J., Anagnostakis, S. L., Milgroom, M. G., and Hillman, B. I. 1994. Isolation and characterization of a virus-resistant mutant of Cryphonectria parasitica. Curr. Genet. 26:528-534.

Powell, W. A., and Van Alfen, N. K. 1987. Two nonhomologus viruses of Cryphonectrica (Endothia) parasitica reduce accumulation of specific virulence-associated polypeptides. J. Bacteriol. 169:5324-5326.

Puhalla, J. E., and Anagnostakis, S. L. 1971. Genetics and nutritional requirements of Endothia parasitica. Phytopathology 61:169-173.

Rostagno, L., Prodi, A., and Turina, M. 2010. Cpkk1, MAPKK of Cryphonectria parasitica, is necessary for virulence on chestnut. Phytopathology 100:1100-1110

Sambrook, J., and Russell, D. W. 2001. Molecular Cloning: A Laboratory Manual. Cold Spring Harbor Press, New York.

Segers, G. C., Zhang, X., Deng, F., Sun, Q., and Nuss, D. L. 2007. Evidence that RNA silencing functions as an antiviral defense mechanism in fungi. Proc. Natl. Acad. Sci. USA 104:12902-12906.

Shapira, R., Choi, G. H., and Nuss, D. L. 1991. Virus-like genetic organization and expression strategy for a double-stranded RNA genetic element associated with biological control of chestnut blight. EMBO J. 10:731-739.

Shi, L., Li, R., Liao, S., Bai, L., Lu, Q., and Chen, B. 2014. Prb1, a subtilisinlike protease, is required for virulence and phenotypical traits in the chestnut blight fungus. FEMS Microbiol. Lett. 359:26-33.

So, K. K., Ko, Y. H., Chun, J., Kim, J. M., and Kim, D. H. 2017. Mutation of the Slt2 ortholog from Cryphonectria parasitica results in abnormal cell wall integrity and sectorization with impaired pathogenicity. Sci. Rep. 7: 9038.

Sun, Q., Choi, G. H., and Nuss, D. L. 2009. Hypovirus-responsive transcription factor gene prol of the chestnut blight fungus Cryphonectria parasitica is required for female fertility, asexual spore development, and stable maintenance of hypovirus infection. Eukaryot. Cell 8:262-270.

Toei, M., Saum, R., and Forgac, M. 2010. Regulation and isoform function of the V-ATPases. Biochemistry 49:4715-4723.

Van Alfen, N. K., Jaynes, R. A., Anagnostakis, S. L., and Day, P. R. 1975. Chestnut blight: Biological control by transmissible hypovirulence in Endothia parasitica. Science 189:890-891.

Wang, J., Lu, L., Yang, Y., Chen, Q., and Chen, B. 2014. Proteomic analysis of Cryphonectria parasitica infected by a virulence-attenuating hypovirus. Wei. Sheng. Wu. Xue. Bao. 54:803-812.

Wang, J., Wang, F., Feng, Y., Mi, K., Chen, Q., Shang, J., and Chen, B. 2013. Comparative vesicle proteomics reveals selective regulation of protein expression in chestnut blight fungus by a hypovirus. J. Proteomics 78: 221-230.

Xie, Y., Coukell, M. B., and Gombos, Z. 1996. Antisense RNA inhibition of the putative vacuolar $\mathrm{H}(+)$-ATPase proteolipid of Dictyostelium reduces intracellular $\mathrm{Ca}^{2+}$ transport and cell viability. J. Cell Sci. 109:489-497.

Yao, Z., Zou, C., Zhou, H., Wang, J., Lu, L., Li, Y., and Chen, B. 2013. A(1)pyrroline-5-carboxylate/glutamate biogenesis is required for fungal virulence and sporulation. PLoS One 8:e73483.

Zhu, J., Zhu, X. G., Ying, S. H., and Feng, M. G. 2017. Effect of vacuolar ATPase subunit $\mathrm{H}(\mathrm{VmaH})$ on cellular $\mathrm{pH}$, asexual cycle, stress tolerance and virulence in Beauveria bassiana. Fungal Genet. Biol. 98:52-60. 